\title{
O SONO COMO ATIVADOR DO ELETRENCEFALOGRAMA NOS PACIENTES EPILÉTICOS
}

\author{
Rosa Helena Longo* \\ José Geraldo Camargo Lima*
}

\begin{abstract}
Desde que em 1947 os Gibbs $^{8}$ chamaram a atenção para a ação do sono como ativador de descargas epilépticas no eletrencefalograma, têm sido esse método amplamente utilizado. Naquele trabalho esses autores mostraram que o sono duplicava o número de traçados patológicos em casos de epilepsia sintomática e triplicava esse número em casos de epilepsia idiopática; além disso admitiram que os focos em vigília não eram obliterados durante o sono e realçaram o valor do sono nas crises psicomotoras, nas quais a percentagem de traçados anormais se elevou de 30 para 90\%. Trabalhos ulteriores como os de Bado \& Dies ${ }^{1}$, Batini \& col. ${ }^{2}, 3$, Caveness ${ }^{4}$, Caveness \& col. ${ }^{5}$, Delange \& col. ${ }^{6}$, Gastaut \& col. $^{7}$, Kajtor ${ }^{11}$, Kellaway ${ }^{12}$, Passouant ${ }^{15}$, Silverman ${ }^{17}$, Silverman \& Morisaki ${ }^{18}$ e White \& col. ${ }^{19}$ confirmaram ser o sono bom sensibilizador das descargas epilépticas. Discordando desses resultados temos Gloor \& col. ${ }^{9,10}$, Merlis \& col. ${ }^{13}$ e Nekhorocheff ${ }^{14}$. Entre nós Pupo \& Reis Filho ${ }^{16}$, analizando os dados assinalados em 700 pacientes epilépticos mostraram que a freqüência de eletrencefalogramas anormais foi mais alta em vigilia $(50 \%)$ do que em estado de sono (26\%). Essas discordâncias encontradas na literatura nos levaram à feitura deste trabalho.
\end{abstract}

\section{MATERIAL, MÉTODO E RESULTADOS}

O material consta de 1.868 pacientes epilépticos* nos quais foram feitos eletrencefalogramas em virgília (repouso e hiperpnéia) e em sono (fase lenta), sendo os achados comparados. Foi considerado que houve ativação quando o sono, por si só, provocou o aparecimento de anormalidades eletrencefalográficas que não existiam antes ou quando aumentou de modo indubitável as alterações registradas em vigília; consideramos como desativação quando as anormalidades registradas em vigilia desapareceram durante o sono ou diminuiram de modo indubitável. Os tipos clínicos da sindrome convulsiva apresentada pelos pacientes e a ação do sono estão registrados na tabela 1. Não encontramos diferença significativa entre os achados durante o sono espontâneo e o medicamentoso.

Trabalho do Departamento de Neurologia e Neurocirurgia da Escola Paulista de Medicina: * Professores Adjuntos.

* Nota dos autores - Este material fez parte do relatório apresentado por um de nós (R.H.L.) - Eletrencefalografia e sono - ao II Congresso Pan-Americano de Neurologia (Porto Rico, 22-28 outubro, 1967). 


\begin{tabular}{lccc}
\hline & & \multicolumn{2}{c}{ Ação do sono } \\
Síndrome convulsiva & N.o de casos & $\begin{array}{c}\text { Ativação } \\
\text { Desativação } \\
\%\end{array}$ \\
\hline \hline Crises generalizadas & & 11 & 17 \\
Temporais não P.S.M. & 572 & 10 & 21 \\
Psicomotoras (P.S.M.) & 121 & 26 & 20 \\
Focais não temporais & 118 & 13 & 21 \\
Crises convulsivas noturnas & 410 & 15 & 8 \\
Crises convulsivas febris & 314 & 7 & 11 \\
Crises convulsivas + retardo psicomotor & 165 & 168 & \\
\end{tabular}

Tabela 1 - Tipos clínicos da síndrome convulsiva e ação do sono como ativador e desativador.

\section{COM E N TÁRIOS}

$\mathrm{Na}$ maioria de nossos pacientes o eletrencefalograma realizado durante o sono apenas confirmou os achados encontrados em vigilia, quer nos traçados normais como nos anormais. Concordando com Gloor \& col. ${ }^{9}, 10$, Merlis $\&$ col. ${ }^{13}$ e Nekhorocheff ${ }^{14}$, a ação ativadora do sono foi registrada em número relativamente pequeno de casos, tendo atingido o seu máximo no grupo de pacientes com crises psicomotoras $(26 \%)$. Deve ser realçado o fato de que entre os pacientes com crises generalizadas, crises temporais não psicomotoras, crises focais não temporais e crises convulsivas noturnas o sono atuou mais como desativador do que ativador das anormalidades registradas em vigília, o que contraria a idéia de Gibbs e Gibbs ${ }^{8}$.

Em conclusão, pensamos que o papel ativador do sono sobre as anormalidades eletrencefalográficas encontradas nos pacientes epilépticos não deve ser muito valorizado pois não raramente essas alterações são muito atenuadas ou mesmo bloqueadas totalmente nesse estado.

\section{R E S U M O}

Visando ao estudo da ação do sono como ativador do eletrencefalograma em pacientes epilépticos, foram estudados 1.868 pacientes com sindromes convulsivas $(572$ com crises generalizadas, 121 com crises temporais não psicomostras, 118 com crises psicomotoras, 410 com crises focais não temporais, 314 com crises noturnas, 165 com crises febris, 168 com crises convulsivas associadas a retardo psicomotor). Em todos os pacientes foram feitos eletrencefalogramas em vigília (repouso e hiperpnéia) e durante o sono (fase lenta) sendo os achados comparados. Em nosso material não encontramos diferença significativa de resposta entre o sono espontâneo e o medicamentoso. Na maioria de nossos pacientes o traçado realizado durante o sono confirmou os achados registrados em vigilia. A ação ativadora do sono foi evidenciada em um número relativamente pequeno de casos, tendo atingido o 
seu máximo no grupo de pacientes com crises psicomotoras (26\%). Nas crises generalizadas, temporais não psicomotoras, focais não temporais e noturnas o sono funcionou mais como desativador do que como ativador das anormalidades registradas em vigília.

\section{S U M M A R Y}

\section{The sleep as an activator on epileptic patient's electroencephalogram}

In order to study the influence of sleep on the electroencephalograms of epileptic patients the tracings obtained of 1.868 such patients were analised (572 generalized seizures; 121 non-psychomotor temporal; 118 psychomotor; 410 non-temporal focal; 314 noctural seizures; 165 febrile seizures; 168 convulsive seizures associated with psychomotor retard).

The electroencephalograms were made with the patients awake (rest and hyperpnea) and sleeping (slow phase), the findings being compared. There was not found any significant difference between the EEG tracings obtained during spontaneous sleep and medically induced sleep. In the majority of the cases the records obtained while the patients were asleep merely confirmed the ones made while the patients were awake. The activator action of sleep appeared only in small number of the cases, having it's peak on the group with psychomotor seizures $(26 \%)$. In the generalized non-psychomotor temporal, non-temporal focal and noctural seizures the sleep acted as an antiactivator instead of an activator of the abnormalities recorded while the patients were awake.

\section{REFEREN C I A S}

1. BADO, J. V. \& DIES, J. M. S. - El sueno inducido como metodo de elección en la activación del EEG de los epilepticos. Electroenceph. clin. Neurophysiol. 5:460, 1953.

2. BATINI, C.; FRESSY, J.; NAQUET, R.; ORFANOS, A. \& SAINT-LAURENT, J. - Etude du sommeil nocturne chez 20 sujets présentant descharges irritatives focalisées. Rev. neurol. (Paris) 108:172, 1963.

3. BATINI, C.; CRITICOS, A.; FRESSY, J. \& GASTAUT, H. - Nocturnal sleep in patients presenting epilepsy with bisynchronous EEG discharges. Electroenceph. clin. Neurophysiol. 14:957, 1962.

4. CAVENESS, W. F. - Relations of seizures to various aspects of sleep. Electroenceph. clin. Neurophysiol. 9:380, 1957.

5. CAVENESS, W. F.; ROBBINS, M. B. \& JOHNS, T. R. - Convulsive seizures coincident with sleep with special enphasis on the relation between seizure and changes in phase of sleep. Electroenceph. clin. Neurophysiol. 10:182, 1958.

6. DELANGE, M.; CASTAN, P. H.; CADIlHAC, J. \& PASSOUANT, P. — Study of the night sleep during centrencephalic and temporal epilepsies. Electroenceph. clin. Neurophysiol. 14:777, 1962.

7. GASTAUT, H.; BATINI, C.; FRESSY, J.; BROUGHTON, R.; TASSINARI, C. A. \& VITTINI, F. - Étude électroencephalographique des phénomènes épisodiques épileptiques au cours du sommeil. In Le Sommeil de Nuit Normal et Pathologique. Vol. 2 da série Electroencephalographie et Neurophysiologie Clinique. Masson et Cie., Paris, 1965. 
8. GIBBS, E. L. \& GIBBS, F. A. - Diagnostic and localising value of electroencephalographic studies in sleep. Nerv. Ment. Dis. Proc. 26:366, 1947.

9. GLOOR, P.; TSAI, C. \& HADDAD, F. - An assessment of the value of sleep electroencephalography for the diagnosis of temporal lobe epilepsy. Electroenceph. clin. Neurophysiol. 10:663, 1958.

10. GLOOR, P. TSAI, C.; HADDAD, F. \& JASPER, H. H. - The lack of necessity for sleep in the EEG or EEG diagnosis of temporal seizures. Electroenceph. clin. Neurophysiol. 9:379, 1957.

11. KAJTOR, F. - The influence of sleep and the waking state on the epileptic activity of different cerebral structures. Epilepsia (Amsterdam) 3:274, 1962.

12. KELLAWAY, P. - The use of sedative-induced sleep as an aid to electroencephalographic diagnosis in children. J. Pediat. 37:862, 1950.

13. MERLIS, J. K.; GROSSMAN, C. \& HENRIKSEN, G. F. - Comparative effectiveness of sleep and metrazol-activated electroencephalography. Electroenceph. clin. Neurophysiol. 3:71, 1951.

14. NEKHOROCHEFF, I. - Le valeur du sommeil en tant que méthode de sensibilisation EEG chez l'enfant. Rev, neurol. (Paris) 83:570, 1950.

15. PASSOUANT, P. - Sémiologie électroencephalografique du sommeil normal et pathologique. Rev. neurol. (Paris) 83:545, 1950.

16. PUPO, P. P. \& REIS FILHO, I. - Convulsion in childhood: electroencephalographic aspects. Arq. Neuro-Psiquiat. (São Påulo) 25:17, 1967.

17. SILVERMAN, D. - Sleep as a general activation procedure in electroencephalography. Electroenceph. clin. Neuropysiol. 8:317, 1956.

18. SILVERMAN, D. \& MORISAKI, A. - Re-evaluation of sleep electroencephalography. Electroenceph. clin. Neurophysiol. 10:425, 1958.

19. WHITE, P.; DYKEN, M.; GRANT, P. \& JACKSON, L. - Electroencephalographic abnormalities during sleep as related to the temporal distribution of seizures. Epilepsia (Amsterdam) 3:167, 1962.

Departamento de Neurologia e Neurocirurgia - Escola Paulista de Medicina - Caixa Postal 5496 - 01000 São Paulo, SP - Brasil. 